\title{
Report
}

\section{Vizepräsidentin Viviane Reding fordert einen neuen Datenschutzpakt für Europa}

Vor zwei Jahren hatte die Europäische Kommission einen Vorschlag für eine umfassende Reform der EU-Datenschutzvorschriften vorgelegt, um diese an die Erfordernisse des 21. Jahrhunderts anzupassen (IP/12/46). Seitdem konnten bereits beträchtliche Fortschritte erzielt werden'1. Im Oktober 2013 sprach sich der federführende Ausschuss des Europäischen Parlaments - der Ausschuss für bürgerliche Freiheiten, Justiz und Inneres (LIBE) - mit großer Mehrheit für die Vorschläge der Kommission aus (MEMO/13/923). Im Rat (Justiz) sind bereits zahlreiche Diskussionen über die Vorschläge geführt worden, doch bislang konnten sich die Minister nicht auf ein Mandat für die Verhandlungen mit dem Europäischen Parlament einigen. Am 28. Januar 2014, dem Europäischen Tag des Datenschutzes forderte Viviane Reding, Vizepräsidentin der Europäischen Kommission, in einer Rede im Centre for European Policy Studies (CEPS) einen "Datenschutzpakt für Europa"

„Beim Datenschutz ist es Zeit zu handeln. Das Europäische Parlament hat das verstanden und sich im Oktober in seinem LIBE-Ausschuss für eine restriktive Verordnung mit angemessenen Sanktionen ausgesprochen, um die Einhaltung der europäischen Vorschriften zu gewährleisten. In Athen haben sich die drei Organe die Europäische Kommission, die beiden Berichterstatter des Europäischen Parlaments sowie der derzeitige griechische und der künftige italienische Ratsvorsitz - letzte Woche auf einen Fahrplan geeinigt, um die Datenschutzreform bis Ende dieses Jahres zu verabschieden. Wenn man bedenkt, dass bis zur Annahme der Datenschutzrichtlinie aus dem Jahr 1995 fünf Jahre verhandelt wurde, ist das ein recht gutes Ergebnis. Es steht auch im Einklang mit den Schlussfolgerungen, die die Staats- und Regierungschefs auf ihrem Gipfel vom Oktober letzten Jahres angenommen haben. Darin unterstreichen sie, dass die Datenschutzverordnung bis spätestens 2015 in allen Mitgliedstaaten gelten sollte", so Vizepräsidentin Reding zur Frage des Verhandlungstempos in ihrer Rede.

Außerdem betonte sie: „Die EU kann mit ihren Bemühungen zur Wiederherstellung des Vertrauens nur dann glaubwürdig und Vorbild für andere Kontinente sein, wenn sie das eigene Haus in Ordnung bringt."

Als geeignete Lösung, um das Vertrauen - in die transatlantischen Beziehungen und in den Umgang mit personenbezogenen Daten durch Unternehmen - wiederherzustellen, schlägt Vizepräsidentin Reding einen Datenschutzpakt für Europa vor, der sich auf acht Grundsätze stützen sollte:

- Die Reform des Datenschutzes muss rechtlich verankert werden. Wir müssen den Gesetzgebungsprozess daher 2014 mit Nachdruck vorantreiben.

- Bei der Reform sollte nicht zwischen privatem und öffentlichem Sektor unterschieden werden. In Zeiten, in denen der öffentliche Sektor personenbezogene Daten erfasst, verarbeitet und teils

1 MEMO/14/60 vom 27. Januar 2014: http://europa.eu/rapid/ press-release_MEMO-14-60_en.htm sogar verkauft, wäre dies für die Bürgerinnen und Bürger einfach nicht nachvollziehbar. Zudem sind solche Unterscheidungen oft schwierig, beispielsweise wenn lokale Behörden Speicherplatz bei privaten Cloud-Anbietern anmieten.

- Gesetze, die Datenschutzregeln festschreiben oder die die Privatsphäre betreffen, müssen öffentlich diskutiert werden, da sie sich auf die Wahrung der Bürgerrechte im Internet auswirken.

- Datenerfassung sollte zielgerichtet und auf ein Maß beschränkt sein, das im angemessenen Verhältnis zu den vorgegebenen Zielen steht. Eine flächendeckende Überwachung der elektronischen Kommunikation ist nicht akzeptabel.

- Die Rechtsvorschriften müssen klar und deutlich sein und auf dem neuesten Stand gehalten werden. Es kann nicht angehen, dass Staaten moderne Überwachungsprogramme auf Grundlage veralteter, in einem anderen technischen Zeitalter verfasster Regeln entwickeln.

- Das Argument der nationalen Sicherheit sollte sparsam verwendet werden. Es sollte nicht zur Regel werden, sondern die Ausnahme bleiben.

- Eine wirkliche Aufsicht kann es nur mit Einbeziehung der Gerichte geben. Eine Aufsicht durch die Exekutive ist gut. Eine Aufsicht durch die Parlamente ist notwendig. Die Aufsicht durch die Gerichte ist unerlässlich.

- Eine Botschaft an unsere amerikanischen Freunde: Datenschutzvorschriften sollten unabhängig von der Staatsangehörigkeit der betreffenden Person angewandt werden. Unterschiedliche Standards für Inländer und Ausländer anzuwenden, ergibt angesichts der Offenheit des Internets keinen Sinn.

\section{Statement von Bundesinnenminister Dr. Thomas de Maizière: Der Europäische Datenschutztag steht im Zeichen der Datenschutzreform}

Das europäische Datenschutzrecht stammt aus dem Jahre 1995. Damals war noch nicht vorhersehbar, dass das Internet von zentraler Bedeutung für die Lebensführung der Bevölkerung werden würde. Die Hauptakteure des Internets sind private, global agierende Unternehmen wie Google, Facebook, Yahoo, Twitter, usw. Sie sammeln Datenmengen in damals noch unvorstellbarem Ausmaß. 1995 wurden diese Datenmengen noch in Kilo- und Megabyte gemessen. Heute zählen wir sie in Yottabyte. Das ist eine Zahl mit 24 Nullen. Außerdem entwickeln sich die technischen Möglichkeiten zur Auswertung und Verknüpfung von Daten rasant. Der Begriff „Big Data“ steht für die technischen Möglichkeiten, in unglaublich großen Datenmengen Muster für das Verhalten von Menschen zu erkennen und mit Hilfe von Algorithmen Verhaltensprognosen zu errechnen. Private, global tätige Unternehmen sind hier längst an staatlichen Akteuren vorbeigezogen, und zwar allein aus Geschäftsinteresse. Das fordert neue Schutzkonzepte heraus. Das 